\title{
El enfoque epistemológico de la teoría crítica y su actualidad
}

\author{
Critical theory epistemological perspective and its actuality
}

María Luz Ruffini (ruffiniluz@gmail.com) Facultad de Ciencias Sociales, Universidad Nacional de Córdoba (Córdoba, Argentina) ORCID: 0000-0002-4101-2396

\begin{abstract}
In this paper, we discuss the epistemological approach -as defined by J. Padrón- called critical theory. In the first part, we explain its ontological dimension through the category of totality, as T. Adorno and M. Horkheimer develop it throughout their works, in opposition to Popperian empiricism. In the following section, we analyse the gnoseological dimension of critical theory around the political in scientific production, using as a driving axis the debate between $\mathrm{K}$. Popper and T. Adorno with special emphasis on the link between science and praxis, the production of scientific knowledge and a critical and transformational intentionality of the social world, which constitutes a partially meta-theoretical basis for this approach. Finally, in the conclusions, we explain some central implications for the current social research: the increasing fragmentation and hyper specialization of the scientific field against the ontological notion of totality, and the need to follow the critical construction of knowledge with an adequate meta-critical theoretical and practical position of the researcher.
\end{abstract}

Key words: critical theory, totality, praxis, positivism, metacritical.

\section{Resumen}

En este trabajo se presenta un abordaje explicativo del enfoque epistemológico -tal como es definido por J. Padrón- propio de la corriente de pensamiento convencionalmente denominada teoría crítica. En la primera parte abordamos su dimensión ontológica a través de la categoría de totalidad, tal como T. Adorno y M. Horkheimer la desarrollan a lo largo de sus trabajos, en oposición al empirismo popperiano. En la sección siguiente damos cuenta de la dimensión gnoseológica de la teoría crítica, desarrollando las reflexiones y discusiones en torno a lo político en la producción científica y empleando como eje conductor el debate entre K. Popper y T. Adorno, con especial énfasis en el vínculo entre ciencia y praxis, entre producción de conocimiento científico e intencionalidad crítica y transformadora del mundo social, lo cual constituye un fundamento parcialmente meta-teórico clave para este enfoque. Finalmente, en las conclusiones, explicitamos algunas implicancias de lo trabajado para la investigación social actual: la centralidad de oponer a la creciente fragmentación e hiperespecialización del campo científico la noción ontológica de totalidad y la necesidad de acompañar la construcción crítica del conocimiento con una adecuada metacrítica de la posición teórica y práctica del investigador.

Palabras clave: teoría crítica, totalidad, praxis, positivismo, metacrítica. 


\section{Introducción}

No es posible, si se pretende encarar con seriedad la labor científica de investigación, eludir el análisis de sus fundamentos más profundos, los cimientos sobre los cuales se erige toda construcción teórica y cualquier abordaje empírico. En este sentido explicitar cuestiones epistemológicas -es decir, vinculadas con las vías legítimas de producción y validación del conocimiento, en este caso, científico- es una tarea ineludible, pues sitúa la labor en una tradición y permite ponderar de modo más acabado su consistencia y potencial.

En los últimos años, es posible reconocer en el campo de las ciencias sociales la preeminencia de un consenso epistemológico de tipo post-empirista, en franco contraste con el denominado "consenso ortodoxo", de corte positivista y tradicionalmente identificado con el llamado Círculo de Viena. Según J. Alexander, esta corriente epistemológica puede ser caracterizada en base a cuatro postulados fundamentales: la ruptura epistemológica radical entre proposiciones empíricas y no empíricas, la certeza de que las cuestiones generales y abstractas no son fundamentales para una disciplina de orientación empírica; la verificación como criterio de evaluación de las proposiciones -lo que iría de la mano con una concepción inductiva de la teoría-, y la creencia en un desarrollo de la ciencia lineal y acumulativo. Por supuesto, esta concepción ha sufrido fuertes cuestionamientos y profundas críticas, a pesar de lo cual sigue manteniendo un carácter hegemónico en el campo científico: "Si bien estos cuatro postulados todavía reflejan con exactitud la opinión común de la mayoría de los científicos sociales -especialmente en Norteamérica-, la nueva tendencia de la filosofía, historia y sociología post-positivista de la ciencia natural surgida a lo largo de las dos últimas décadas los ha criticado abiertamente" (Alexander 1990:6).

Ahora bien, es claro que las posiciones desarrolladas en contraste con esta perspectiva distan de ser homogéneas y han generado, a lo largo del siglo XX, fuertes controversias dentro de la comunidad científica. A fin de dar cuenta de ellas, consideramos valiosa la propuesta de J. Padrón que, a fin de abordar los filtros preteóricos o precognitivos que condicionan los procesos de conocimiento, propone un aparato conceptual capaz de generar una lectura diacrónica y metateórica que trasciende autores y perspectivas particulares, permitiendo construir un "mapa epistemológico" de la ciencia en base a relativamente pocos principios generales variables.

En este sentido, el autor desarrolla una hipótesis basada en el concepto de "enfoques epistemológicos", según la cual "las variaciones observables en los procesos de producción científica obedecen a determinadas convicciones acerca de qué es el conocimiento y de sus vías de producción y validación, sistemas que tienen un carácter preteórico, ahistórico y universal, denominados 'enfoques epistemológicos'. Las variaciones observables generadas por estos enfoques pueden estandarizarse en 'paradigmas', los cuales tienen lugar a lo largo de la historia de la ciencia y se suceden unos a otros en el control de los estándares científicos de las épocas" (Padrón 2007:5).

Sobre esta base, propone un aparato conceptual -en base a la tesis de los "tres mundos" de Popper, asimilable al anterior "triángulo de Odgens"- que contempla dos variables, cada una de las cuales puede adoptar, simplificadamente, claro está, dos valores. La primera de ellas, variable gnoseológica, se define entre el empirismo y el racionalismo; mientras que la segunda, variable ontológica, se dirime entre el idealismo y el realismo. El cruce entre ambas lleva a cuatro cuadrantes y cuatro enfoques epistemológicos hipotéticos, cada uno de los cuales permite dar cuenta de los principales fundamentos presuposicionales a partir de los cuales se conforman los procesos científicos. 
Desde esta perspectiva, entonces, es posible efectuar una lectura diacrónica del siglo XX como momento de génesis de los principales paradigmas epistemológicos, que sentarán las bases de los desarrollos científicos ulteriores. En efecto, el siglo inicia con la hegemonía de la llamada "concepción heredada" y el empirismo del círculo de Viena -empirismo realista-, puesto luego en jaque por el falsacionismo popperiano, a partir del cual cobra fuerza la vertiente teoricista que, con el mayor énfasis otorgado a los aspectos culturales e históricos abrirá la puerta a perspectivas de corte empirista idealista, que presentan cierta confluencia con lo que se estaba planteando desde la Escuela de Frankfurt y la Teoría Crítica, como exponente central del enfoque racionalista idealista. Cabe hacer un paréntesis aquí a fin de clarificar que lo que Padrón denomina idealismo se vincula, en rigor, a la preeminencia de constructos teóricos por sobre la profusión de datos empíricos a la hora de construir conocimiento científico, por lo que el expreso distanciamiento de la Teoría Crítica de las premisas idealistas tradicionales, como la relación inmediata entre pensamiento y realidad e, incluso, del concepto idealista de razón hegeliano -que sin embargo, para A. Honneth no deja de alimentar en última instancia la intuición ética de la empresa frankfurtiana-, no afecta nuestra argumentación en ese sentido. Por otro lado, y finalmente, la década de los ' 60 culmina con el fortalecimiento del racionalismo realista, al modo de Schutz.

En este marco, entonces, es que buscaremos profundizar, tomando como base las dos dimensiones analíticamente diferenciables propuestas por Padrón, en la propuesta epistemológica del cuadrante que este autor definiría analíticamente como racionalista-idealista en su versión primera, representada de modo genérico por la Teoría Crítica. Cabe mencionar que empleamos aquí la denominación de "Teoría Crítica" en oposición a "Escuela de Frankfurt", basándonos en la crítica de Lowenthal al empleo del primer término. En efecto, para éste, en un principio, cuando el primer círculo comienza a reunirse en torno al Instituto de Investigación Social y antes del retorno de Adorno y Horkheimer: "La Escuela de Francfort no era una escuela, sino un proyecto colectivo y cooperativo; el proyecto de poner otra vez en marcha y desarrollar la teoría crítica de la sociedad" (Wellmer 1996:240).

Por supuesto, en este marco amplio la multiplicidad de voces de esta corriente presenta diferencias de importancia, no obstante lo cual creemos, con A. Honneth, que es posible encontrar entre ellas un eje común, un principio de unidad: el modelo intelectual de ensamble entre teoría e historia, según el cual el pasado histórico ha sufrido un proceso de deformación producto de una desviación patológica de la razón, ante el cual la teoría no adopta una relación de correspondencia estática y fragmentaria, sino un vínculo negativo que apela, como dice Horkheimer, a la relación consciente de la teoría con la praxis histórica necesaria para transformar esa historia concebida como irracional.

En efecto, las promesas de una sociedad más justa que la ilustración auguraba se vieron trágicamente desmentidas por el desarrollo histórico basado en el modo de producción capitalista, y este desgarro muestra su cariz más trágico en la experiencia fascista. En este marco, la ciencia y el conocimiento tienen un lugar central, ya que si, como dice Honneth, "las circunstancias sociales que constituyen la patología de las sociedades capitalistas tienen la característica estructural de velar precisamente aquellos hechos que serían motivo de una crítica pública particularmente dura" (Honneth 2009:38), una posición epistemológica que no ponga en primer plano una intencionalidad radicalmente transformadora resulta inaceptable, no solo en virtud de razones epistemológicas, sino también y sobre todo, en base a consideraciones ético-políticas.

En efecto, si desde esta perspectiva se asume un poder emancipador de la razón, en tanto la comprensión racional del mundo puede constituir una vía privilegiada para su transformación, la producción de conocimiento debe llevarse adelante de tal manera que permita dar cuenta críticamente de lo profundo 
de las relaciones de dominación y las razones de su perpetuación. Sobre esta base se define, entonces, la perspectiva epistemológica de esta corriente, que caracterizaremos, siguiendo a Padrón, en función de sus dimensiones ontológica y gnosológica -por supuesto, solo analíticamente diferenciables- teniendo siempre presente, en tanto fundamento parcialmente meta teórico, la dimensión axiológica, a través de la cual se observa la relación -honda, intrínseca, inherente- de esta teoría con la posibilidad de una praxis emancipatoria.

Es interesante, en este sentido, observar cómo al intentar fundamentar la elección de esta temática nos sumergimos, de lleno y sin escalas, en el problema que queremos abordar. En efecto, podemos argumentar sobradamente la centralidad que la particular concepción epistemológica frankfurtiana adquiere para pensar dimensiones de suma relevancia en la producción de conocimiento sobre el mundo social e incluso sustentar esta decisión en el hecho de que los desarrollos epistemológicos más recientes las llamadas "nuevas epistemologías"-, implicaron la profundización de problemas clave para la Teoría Crítica, como "el externalismo o la influencia de factores socio contextuales en los procesos científicos, la inclusión del sujeto y de los actores en los procesos de búsqueda y la interacción sujeto-objeto" (Padrón 2007:15). No obstante, aun el desarrollo más exhaustivo en torno a estas cuestiones no puede eludir una certeza fundamental, en la que la teoría crítica abrevará y sostendrá, también, su edificio teórico y epistémico: una apuesta política contra lo ineluctable del mundo, contra una ciencia incapaz de elevarse más allá de lo dado para brindar las herramientas de una crítica que aspire a ser praxis transformadora.

\section{Dimensión ontológica: la noción de totalidad}

¿Cómo entiende la Teoría Crítica a la realidad social? ¿Cómo debe ser concebida para su abordaje científico, como vimos, con una intencionalidad última de transformación? M. Horkheimer en Historia y Psicología aboga por una filosofía de la historia que, de modo hegeliano -si bien críticamente, en tanto percibe la impotencia de Hegel para pensar la posibilidad de una praxis transformadora-, se ocupe del "conocimiento de la estructura unitaria y dinámica dilucidable en la desconcertante multiplicidad del acontecer" (Horkheimer 2003:25). En este sentido, los hechos históricos solo adquieren su cabal sentido al ser entendidos como parte del despliegue de una totalidad.

Cabe salir al encuentro, en este punto, a la suspicacia de corte empirista, explicitando que el reconocimiento de la centralidad de la categoría de totalidad no sustituye en esta perspectiva a las investigaciones concretas, ni puede ser elevada a la categoría de un poder que necesariamente haría discurrir a la historia por carriles preestablecidos: el todo social no tiene vida propia más allá de los componentes que aúna y de los que consta.

En este sentido, como Adorno pone de manifiesto en su extensa introducción al libro colectivo La disputa del positivismo en Alemania, no hay lugar para dogmatismos deterministas: el todo social se produce y reproduce en virtud de sus momentos particulares, a los que no deja de configurar y determinar, aunque tal determinación no pueda ser probada a partir de la verificación de datos aislados. En sus palabras: "Es innegable que no hay experimento capaz de probar fehacientemente la dependencia de todo fenómeno social respecto de la totalidad, en la medida en que el todo, que preforma los fenómenos tangibles, jamás resultará aprehensible mediante métodos particulares de ensayo. $Y$, sin embargo, la dependencia del hecho o elemento social sometido a observación respecto de la estructura global tiene una validez mucho más real que la de tales o cuales datos verificados -aisladamente- de manera irrefutable y es, desde luego, todo menos una enloquecida elucubración mental" (Adorno 1972:129). La definición de teoría que ofrece Horkheimer en el famoso ensayo Teoría tradicional y teoría crítica asume, en este mismo sentido, la 
necesaria complementariedad entre teoría y empiria, entendiendo que la primera debe asumirse como una hipótesis en relación a lo segundo.

Así, es claro que esta perspectiva lejos está de aislarse de la experiencia o no tomar en consideración a los hechos. Ella asume el carácter mediado de lo particular por la totalidad social: en los fenómenos sociales se expresa la totalidad, y aprehenderla es el objetivo por excelencia de la labor científica. Los fenómenos inmediatamente aprehensibles, en este esquema, adquieren su lugar e importancia relativa en función de la estructura explicativa general. En palabras de Adorno: "Incluso categorías tan alejadas de la inmediatez como la de la sociedad, no pueden ser pensadas sin incluir un inmediato; quien no comience por percibir en los fenómenos sociales lo social que en ellos se expresa, no podrá acceder a un auténtico concepto de sociedad. Pero el momento de la inmediatez ha de ser superado en el proceso del conocimiento" (Adorno 1972:67).

La concepción contraria, denominada "positivista" por los representantes de esta corriente, que si bien incluía "tanto el método cientificista de la fenomenología, de los empiristas, como así también de los epígonos del nominalismo [...] al igual que la hermenéutica filosófica" (Geyer 1985:36) aunque aquí abordaremos sobre todo desde el empirismo popperiano, implica asumir que los hechos pueden ser explicados en base a su mera autorreferencialidad, incurriendo de este modo no solo en un error epistemológico fundamental, sino acarreando también consecuencias políticas de importancia. En efecto, una tarea científica que se reduzca a dar cuenta de lo fáctico, lo dado, sin referir a lo que hay en ellos que los excede y que no puede concebirse más que como la totalidad social de la que son expresión y a la cual contribuyen a configurar, supone rendirse a la ideología dominante y, por tanto, contribuir a la perpetuación de las estructuras de poder y dominación hegemónicas en el momento histórico considerado.

En este marco, el "positivismo" yerra el camino al buscar la objetividad sin la pretensión de construir teóricamente el edificio conceptual que permita sustentar las relaciones entre los hechos sociales particulares. De este modo, lejos de lograr lo que pretende, otorga arbitrariamente carácter objetivo a una particularidad socialmente condicionada, con el agravante de no reconocerla como tal. En este sentido, la ciencia positiva hipostasia el logos burgués-racional-instrumental, elevándolo a categoría universal y transhistórica, al modo de la economía clásica y el sujeto racional. De hecho, la crítica inmanente vinculada a principios lógico formales da por sentado que aquella es la lógica que corresponde a la ciencia y que en base a ella debe evaluarse y criticarse -científicamente hablando- el conocimiento producido. En palabras de Adorno: "Los conceptos supuestamente originarios, y en particular los de la teoría del conocimiento, se hallan mediados en sí mismos, y por eso se hace necesaria una "metacrítica», como esfuerzo del concepto que indica que no hay un primero absoluto, un en sí inmediato e irreductible. Lo primero y lo inmediato, en cuanto conceptos, siempre se hallan mediados, de ahí que no sean lo primero" (Adorno 1986:10).

En contraste, si bien la teoría crítica asume que la práctica científica, como toda praxis, se encuentra social e históricamente configurada, ello no implica que deba contentarse con dar cuenta de sus determinaciones sociológicas. Como Horkheimer plantea en La función social de la filosofía, es posible -y, de hecho, imperativo- que ésta se eleve de dicha particularidad a la universalidad histórica -la totalidad social- a partir de las cuales se conforman dichas propiedades sociológicas.

Ahora bien, esta totalidad fundamental es concebida, en el marco de los aportes del marxismo, como la estructura del modo de producción capitalista, como dice Adorno, la totalidad social se realiza 
concretamente en el sometimiento necesario de los sujetos a la ley abstracta del cambio. En este sentido, la sociedad se concibe como una totalidad real, solo aprehensible a través de la reconstrucción conceptual y con un carácter fundamentalmente contradictorio, por lo cual estructura relaciones sociales irreductibles a un continuo lógico expresado en la facticidad. Es en este marco que es posible comprender por qué Wellmer reconoce en el posmodernismo de Lyotard un retroceso desde Adorno al positivismo, lo que culmina en un conductismo deshistorizado: "En Lyotard, para ser precisos, al entrar en escena la voluntad 'en el sentido de querer lo que se puede' en lugar de la actitud, regulada por la artificialidad y las construcciones de la representación, el postmodernismo se vuelve indiscernible del conductismo [...] el postmodernismo se torna ideología de las posthistoria" (Wellmer 1993:56).

De este modo, vemos que la totalidad no resulta una categoría afirmativa, sino crítica: al aprehender su verdad se devela lo contradictorio e irracional del mundo social, y el consecuente carácter imperativo de su crítica. Y ésta, entendida como un comportamiento humano que tiene por objeto la transformación de la totalidad social, sin conformarse con una reforma parcial, para lo cual baste con recordar la demoledora crítica de Benjamin a las intenciones reformistas de tipo socialdemócrata, entendiéndolas como cautivas de una idea de progreso refractaria a la ruptura revolucionaria de la historia basadas en "La idea de un progreso del género humano en la historia, inseparable de la representación de su movimiento como un avanzar por un tiempo homogéneo y vacío" (Benjamin 2007:34).

La crítica así entendida encuentra, entonces, su fundamento en la historización de esa totalidad, que permite entenderla como producto contingente de la praxis humana, susceptible por tanto de ser modificada. Ello no implica, desde luego, que en la sociedad capitalista la estructura social total no se muestre efectivamente como una instancia inhumana, mecánica, que se impone a los hombres (al modo durkheimiano de los hechos sociales), no obstante lo cual, en palabra de Horkheimer: "La construcción del acontecer histórico como el producto necesario de un mecanismo económico contiene, al mismo tiempo, la protesta contra ese orden, originada justamente en ese mecanismo, y la idea de la autodeterminación del género humano, es decir, la idea de un estado tal que, en él, las acciones de los hombres ya no emanen de un mecanismo, sino de sus mismas decisiones" (Horkheimer 2003:259).

\section{Dimensión gnoseológica: la producción de conocimiento científico}

Ahora bien, teniendo presente lo abordado en el apartado anterior, ¿de qué manera se conoce esa totalidad social?, ¿cómo se configura, en base al presupuesto ontológico de totalidad, la tarea científica de construcción de conocimiento? Para comenzar a ensayar algunas respuestas a estas preguntas, es central situar el problema históricamente, entendiendo que la confrontación de la Teoría Crítica con el "positivismo", paradigmáticamente representado por el debate de principios de los '60 entre K. Popper y T. Adorno, constituye, según ciertos análisis, la tercera disputa metodológica central de las ciencias sociales en idioma alemán desde el siglo XIX.

En este sentido, la polémica a la que hacemos referencia no puede ser abstraída de la célebre ruptura introducida por Max Weber en torno a los juicios de valor en 1909, que de hecho configuró y aun configura gran parte de las discusiones en torno a la externalidad y, de la mano con ello, sobre los fines, naturaleza y función de las ciencias sociales. En este sentido, Max Weber plantea, en base al reconocimiento de la centralidad de las ideas de valor en el mundo social -y, por tanto, en el trabajo científico como parte de éste- la necesidad de mantener un riguroso control metodológico para evitar su indebida introducción en la labor de la ciencia. Si bien es innegable la presencia de la dimensión valorativa en numerosas instancias, como la formulación de problemas, la construcción teórica y el establecimiento de causas probables, esta 
debe mantenerse bajo estricto control a fin de lograr la necesaria -y posible, según su enfoque- asepsia valorativa. Para Popper, la idea de objetividad científica se relaciona con la idea Weberiana de la neutralidad valorativa mientras que, para Adorno, la escisión entre objetividad y valor no resulta tan rotunda en Popper como en Weber, algo que también merece una discusión más extensa, en tanto no puede decirse que en Weber la dimensión simbólico valorativa carezca de centralidad para la labor científica.

Ahora bien, volviendo a debate Popper Adorno, vemos que el primero, como representante de la posición autodenominada "racionalismo crítico", sostiene la imposibilidad de una ciencia de la totalidad social, pues solo se pueden conocer, a su juicio, aspectos concretos de la realidad. Esto le lleva a propugnar, en el terreno de la política, la transformación mediante reformas parciales (o como él le llama "la ingeniería social"). Adorno, como representante de la Teoría Crítica, destacará la idea de totalidad a la hora de pensar la realidad social y sostendrá la necesidad de una transformación radical como única manera de superar las contradicciones sociales. Pero profundicemos algo más en esta discusión tan relevante.

\section{Racionalismo popperiano: objetividad y principios lógicos}

Para Popper, el conocimiento no comienza con la percepción, observación o recopilación de datos y hechos, sino con el planteo de problemas, surgidos de la tensión entre el saber y el no saber. Su tesis principal consiste en plantear que el método de las ciencias sociales, al igual que el de las ciencias naturales, implica ensayar posibles soluciones para sus problemas: tentativas de solución sometidas a un estricto control crítico. Este control es para él objetivo: la crítica se sirve de medios lógicos, como la categoría de contradicción. Pero siempre el conocimiento y la justificación resultan provisionales: la validez radica en que haya resistido -hasta el momento- la crítica más aguda posible. En este sentido, las teorías se definen como sistemas deductivos que constituyen un ensayo de explicación, una posible solución -siempre criticable y provisoria- a un problema.

Así, la objetividad científica radica únicamente en la crítica en el marco de la comunidad científica. No es un asunto individual, sino social, en tanto es resultante de la crítica recíproca entre científicos. Para Popper la sociología del conocimiento no ha considerado este criterio de la objetividad científica, limitándose a explicar la no objetividad en función de la posición social del investigador. No obstante, habría categorías sociales (competencia, tradición, instituciones, Estado) que harían posible construir una teoría de la objetividad. En efecto: aunque no podamos justificar racionalmente las teorías -ni siquiera en términos de probabilidad- sí es posible criticarlas racionalmente, y distinguir lo que es mejor de lo que es peor.

La función de la lógica deductiva es ser un órgano de la crítica. En resumen: si todas las premisas son verdaderas y la inferencia es verdadera, entonces la conclusión ha de ser asimismo verdadera; y, en consecuencia, si en una inferencia válida la conclusión es falsa, no es posible en tal caso que todas las premisas sean verdaderas. La lógica deductiva se convierte en la teoría de la crítica racional, en función de dos conceptos clave: aproximación a la verdad y fuerza explicativa, que son puramente lógicas.

Para Popper es posible y necesario distinguir, en la discusión crítica, entre la relevancia, interés y significado de la producción científica con respecto a los problemas de investigación y los problemas extracientíficos. La resonancia de la construcción weberiana resulta aquí muy clara, en palabras del autor: "hay valores positivos y negativos puramente científicos y hay valores positivos y negativos extracientíficos. $Y$ aunque no es posible mantener totalmente separado el trabajo científico de aplicaciones y valoraciones extracientíficas, combatir la confusión de esferas de valor y, sobre todo, excluir 
las valoraciones extracientíficas de los problemas concernientes a la verdad constituye una de las tareas de la crítica de la discusión científica" (Popper 1978:7).

Por supuesto, la crítica de Adorno ante ello resulta demoledora: "Poner la confianza en que gracias a la aceptación de unas reglas de juego destinadas a hacer posible la cooperación lleguen a "sellar un acuerdo", como se diría en términos familiares al Círculo de Viena, posturas muy divergentes, consiguiéndose así el mayor grado posible de objetividad en el conocimiento, equivale a seguir las huellas del anticuado modelo liberal de quienes se sientan en torno a la mesa de las negociaciones para llegar a una solución de compromiso. Las formas de cooperación científica contienen un grado infinito de mediación social. Popper no deja de calificarlas, desde luego, de "asunto social», pero se desentiende de sus implicaciones" (Adorno 1972:41). Pero veamos con mayor detenimiento la posición de la Teoría Crítica a este respecto y su contraste con el modelo Popperiano.

\section{Teoría crítica: juicios de valor, totalidad social contradictoria y crítica emancipatoria}

El eje central de la crítica a Popper radica en el hecho, obvio y reconocido incluso por él mismo -si bien no lo desarrolla ni extrae las consecuencias de ello- de que la pretendida neutralidad valorativa resulta, en realidad, profundamente partidista. En palabras de Adorno: "Con la ensalzada neutralidad científica ocurre lo mismo que con la apatía política, que por su contenido social acaba revelándose como fuertemente política. Desde Pareto, el escepticismo positivista se alía con cualquier poder vigente, incluido el de Mussolini" (Adorno 1972:42).

En efecto, las categorías supuestamente universales y valorativamente neutras no son para Adorno más que las categorías prácticas de la clase burguesa erigidas como universales incuestionados. En este marco, el problema de la disyuntiva ente presencia y ausencia de juicios de valor se encuentra, en su totalidad, mal planteado: la disputa no se da entre presencia o ausencia de juicios de valor en la ciencia, en tanto los ideales cientificistas de objetividad y neutralidad son en sí también valores, al tiempo que es en el ámbito de los impulsos precientíficos donde puede rastrearse el origen de la ciencia. En última instancia, lo que para Adorno resulta indefendible de la propuesta popperiana es la posibilidad de una ciencia objetiva a la cual haya que aproximarse de manera progresiva. Resulta imperativo reconocer las mediaciones sociales de la labor científica, sin absolutizarla ni instrumentalizarla, pues: "En la medida en que el cientificismo se declara unilateralmente por el momento de la unidad entre individuo y sociedad, en honor a la sistemática lógica, y degrada a la categoría de epifenómeno el momento antagonístico inconciliable con dicha lógica, por la fuerza misma de la situación real se convierte en algo falso" (Adorno 1972:31).

Las diferencias entre ambos autores se concretizan, de manera muy clara, en la idea de problema que propugna cada perspectiva. Para Popper, el problema es algo epistemológico, vinculado a una contradicción en el conocimiento presunto. Para Adorno, éste también es algo práctico: "Lo que está en juego es precisamente la validez de tal distinción. Introduciendo en la ciencia una separación radical entre sus problemas inmanentes y los reales, pálidamente reflejados en sus formalismos, lo único que se conseguiría es una auténtica fetichización de la misma" (Adorno 1972:126).

De este modo, la Crítica no puede reducirse a la crítica del método o los juicios sobre el mundo, sino que debe extenderse a la cosa misma: "Cuando la crítica de las categorías sociológicas se reduce a la crítica del método y cuando la discrepancia entre concepto y cosa se produce a costa de la cosa, que no es lo que pretende ser, lo que decide es el contenido del teorema sujeto a crítica. La vía crítica no es meramente formal, sino también material; si sus conceptos han de ser verdaderos, una sociología crítica no puede ser, 
por fuerza -y a tenor de su propia idea-, sino crítica de la sociedad, como Horkheimer razonó en su ensayo sobre la teoría tradicional y la crítica" (Adorno 1972:131). En efecto, para Horkheimer el tratamiento de las cuestiones epistemológicas y metodológicas resulta fundamental: entendiendo al positivismo como manifestación de una razón instrumental y, por tanto, funcional a la dominación colectiva, reconocerá a la teoría la función social de herramienta de develamiento de las estructuras de dominación y, por tanto, contribución a la emancipación. En palabras de Horkheimer: "No hay una teoría de la sociedad, ni siquiera la del sociólogo que generaliza, que no incluya intereses políticos acerca de cuya verdad haya que decidir, ya no mediante una reflexión neutral en apariencia, sino nuevamente actuando y pensando, es decir en la actividad histórica concreta" (2003:253).

\section{Conclusiones}

En momentos en que en el campo científico experimenta un proceso de fragmentación e hiperespecialización, junto con la proliferación de una convicción cuasi religiosa ante las corrientes teóricas hegemónicas, creemos valioso retomar las dimensiones ontológica y gnoseológica que constituyen la propuesta epistemológica de la Teoría Crítica.

En efecto, por un lado, la especialización y la fragmentación de los dominios del conocimiento en torno al mundo social, si bien resultan inevitables en el grado actual de desarrollo del campo científico, no pueden prescindir de la idea de totalidad que la teoría crítica -en tanto heredera del marxismo- recuperó y articuló con otras líneas de trabajo contemporáneas a su producción. En este sentido, es claro que la sociedad supone la estructuración de relaciones de poder interrelacionadas y articuladas, que constituyen el fundamento de los hechos sociales particulares que las investigaciones sociales abordan, y esta consideración incluye a los procesos revolucionarios y contestatarios, que tienen que contar con las relaciones de dominación cristalizadas en un momento histórico determinado. De este modo, toda investigación particular debe asumir su vinculación con esta lógica del todo social, si no quiere caer en un trabajo parcial, fragmentario e incapaz de fomentar una crítica de lo dado.

Por otro lado, el reconocimiento del carácter fundamentalmente valorativo y socialmente condicionado de las teorías y los criterios de cientificidad que las sustentan, siempre producto de un mundo social contradictorio en el que el conflicto es inerradicable, implica ineludiblemente la necesidad de una metacrítica de la propia posición teórica y práctica en el campo de producción de conocimiento junto con la crítica de dicha totalidad social.

En efecto, cuando la labor científica construye conocimiento sobre el mundo social a través del cual se pone en evidencia su carácter injusto, inhumano, opresor (irracional, para la Teoría Crítica), abre la puerta a la praxis, entendida como el pensamiento devenido acción política transformadora de la realidad, en línea con la famosa Tesis 11 sobre Feuerbach: "Los filósofos no han hecho más que interpretar de diversos modos el mundo, pero de lo que se trata es de transformarlo" (Marx 2014:61). Por supuesto, las armas de la crítica no pueden reemplazar a la crítica de las armas, pero es posible que el conocimiento y la teoría se conviertan en armas, al dar cuenta y permitir hacer consciente la dimensión más ignominiosa de la opresión.

Como diría W. Benjamin, corresponde a la ciencia el pensar el presente de tal modo que, lejos de entenderlo como el resultado inevitable de una cadena de acontecimientos progresistas, se lo asuma en tanto resultado contingente de un proceso histórico de barbarie, de muerte, de esclavitud, de injusticia. Pero también es propio de la labor científica reconstruir la esperanza, reconociendo y produciendo 
simultáneamente, por medio de la teoría devenida en instrumento de lucha, las oportunidades revolucionarias en la continuidad del tiempo histórico.

\section{Bibliografía}

Alexander, J. 1990. La centralidad de los clásicos, pp. 6-57. En: A. Giddens y J. Turner. La teoría social hoy. México: Alianza.

Adorno, T. 1972. La disputa del positivismo en Alemania. Barcelona: Grijalbo.

Adorno, T. 1986. Sobre la metacrítica de la teoría del conocimiento. Barcelona: Planeta de Agostini.

Benjamin, W. 2007. Sobre el concepto de historia. Tesis y fragmentos. Buenos Aires: Piedras de papel.

Geyer, C. 1985. Teoría crítica: Max Horkheimer y Theodor W. Adorno. Barcelona: Laia.

Honneth, A. 2009. Patologías de la razón. Historia y actualidad de la teoría crítica. Buenos Aires: Katz editores.

Horkheimer, M. 2003. Teoría crítica. Buenos Aires: Amorrortu.

Marx, K. 2014. Antología. Buenos Aires: Siglo XXI.

Padrón, J. 2007. Tendencias epistemológicas de la investigación científica en el siglo XXI. Cinta moebio 28: 1-32. www.moebio.uchile.cl/28/padron.html

Popper, K. 1978. La lógica de las ciencias sociales. México: Grijalbo.

Wellmer, A. 1993. Sobre la dialéctica de modernidad y postmodernidad. La crítica de la razón después de Adorno. Madrid: Visor.

Wellmer, A. 1996. Finales de partida. La modernidad irreconciliable. Madrid: Cátedra.

Recibido el 24 Feb 2017

Aceptado el 12 May 2017 